\title{
Efectos de una intervención integral en la reducción de factores de riesgo cardiovascular en mujeres con sobrepeso $u$ obesidad de la Región de Los Ríos
}

\author{
LEONORA FUENTES L. ${ }^{\text {aa }}$, ARIEL A. MUNOOZ ${ }^{2 b}$
}

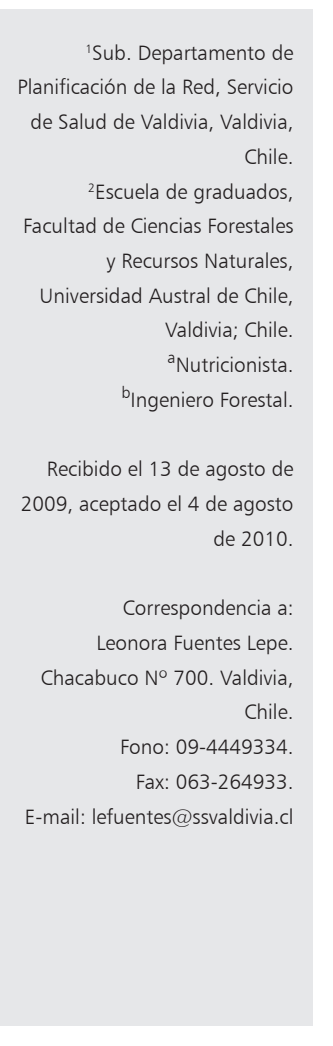

\section{Results of an integral lifestyle modification program to reduce weight among overweight and obese women}

Background: In Chile, the Ministry of Health implemented an integral therapeutic program to reduce cardiovascular risk and increase physical activity (PA) among the population. Aim: To assess the effectiveness of the program in a group of overweight or obese adult women with a family history of Type 2 Diabetes and / or prehypertensive and / or prediabetes. Material and Methods: A group of 128 women aged $35 \pm 10$ years with a mean body mass index (BMI) of $33 \mathrm{~kg} / \mathrm{m} 2$ were invited to participate in a program that lasted four months and included PA sessions and workshops with psychologists and nutritionists. Weight and waist circumference were measured at baseline and at four and six months of follow up. The blood glucose was measured at baseline and at 4 months, only in the affected income. Results: At four months the reduction in weight and waist circumference observed, was around $2 \%$, and at 6 months the reductions was around 3\%. The largest reductions were observed at 6 months in obese patients $\geq 40$ years. Women that were more compliant with $P A$ sessions had a greater weight reduction. The blood glucose levels decreased signifcantly independent of the reduction of anthropometric parameters. Conclusions: The program is considered successful to reduce cardiovascular risk factors and increase the practice of $P A$. The intervention was especially effective in patients $>40$ years possibly due to higher food education, maturity and responsibility to the intervention.

(Rev Med Chile 2010; 138: 974-981).

Key words: Life style; Obesity; Physical exertion.
$\mathrm{E}$ 1 perfil epidemiológico de Chile presenta un predominio de enfermedades crónicas no transmisibles derivadas de la obesidad y el sedentarismo ${ }^{1}$. Esta situación quedó en evidencia en la Encuesta de Salud de 2003 que reveló elevadas cifras de sobrepeso $(37,8 \%)$ y obesidad $(22 \%)$ en adultos $^{2}$. Por su parte, la Encuesta de Calidad de Vida de 2006, señaló que sólo el 8\% de los adultos realiza actividad física (AF), tres o más veces por semana ${ }^{3}$.

Las recomendaciones internacionales, ad- vierten la necesidad de realizar cambios en los estilos de vida (alimentación y actividad física), como medida preventiva básica para disminuir la carga global de enfermedades ${ }^{4}$. Es por ello que en Chile el Ministerio de Salud a través de VIDA Chile $^{5}$, incluyó como desafíos en sus políticas de promoción y objetivos sanitarios al 2010 frenar el sedentarismo, obesidad y sus enfermedades asociadas, para favorecer la reducción de mortalidad por enfermedades cardiovasculares ${ }^{6,7}$.

Diferentes estudios han demostrado que 
cambios positivos en los niveles de AF y hábitos alimentarios, reducen la prevalencia de obesidad y grasa abdominal, siendo este último un factor relevante de riesgo cardiovascular y diabetes mellitus tipo $2^{8}$. Un estudio realizado en EE.UU. en 84.941 enfermeras durante 16 años, concluyó que el $91 \%$ de los casos de diabetes son atribuibles a hábitos de alimentación y $\mathrm{AF}^{9}$. De igual manera, en Finlandia un ensayo realizado en 522 sujetos a los cuales se les intervino durante 3,2 años mediante consejerías en estilos de vida saludables, estableció $58 \%$ de disminución en la incidencia de diabetes ${ }^{10}$.

Bajo este contexto, en Chile el Ministerio de Salud puso en marcha el 2004 un programa piloto basado en la práctica habitual de AF y educación alimentaria para la prevención de enfermedades crónicas en adultos, el cual destacó resultados favorables en la reducción de peso y niveles de glicemia ${ }^{11}$.

Basado en esta experiencia, el 2006 comenzó a implementarse a nivel nacional un programa preventivo-promocional de atención integral para pacientes sobrepeso u obesos con riesgo de padecer enfermedades crónicas no transmisibles. Sus propósitos fueron lograr una baja de peso significativa, considerada sobre el 5\% del peso corporal inicial, reducir el riesgo cardiovascular asociado a diabetes tipo 2 e hipertensión y aumentar los niveles de AF. La intervención consistió en controles de médico, psicólogo y nutricionista, educaciones grupales de alimentación saludable, terapia psicológica y sesiones de AF.

El objetivo del presente estudio fue evaluar los efectos de este programa en la promoción de AF, reducción de peso, IMC (Índice de Masa Corporal), CC (Circunferencia de Cintura) y glicemia, en un grupo de mujeres de cinco comunas de la Región de Los Ríos.

\section{Material y Método}

Se evaluó la aplicación del "Programa de Alimentación Saludable y Actividad Física” (PASAF), en mujeres adultas de la Región de Los Ríos asociadas a riesgo cardiovascular.

\section{Diseño de investigación}

Estudio no experimental, longitudinal de panel en mujeres en control en 5 consultorios de la región durante el año 2008, que ingresaron al Programa PASAF y que cumplieron con todas las evaluaciones.

\section{Sujetos de estudio}

Ingresaron al programa 128 mujeres, $\geq 18$ años con IMC entre 25 y $40 \mathrm{~kg} / \mathrm{m}^{2}$, que cumplieron con al menos uno de los siguientes criterios de ingreso: 2 glicemias en ayunas entre 100 a $125 \mathrm{mg} /$ $\mathrm{dL}$; antecedentes de padre, madre o hermano con diabetes mellitus tipo 2 (DM2); cifras de presión arterial sistólica entre 130-139 mmHg y/o 85-89 $\mathrm{mmHg}$ diastólica.

Sin antecedentes personales de: hipertensión arterial, diabetes nellitus, eventos cardiovasculares, insuficiencia orgánica, patología oncológica.

A todos los pacientes se les informó de la intervención y aceptaron ingresar al programa firmando una carta compromiso. La publicación de los resultados del programa fue aprobado por la dirección del Servicio de Salud Valdivia.

\section{Intervención}

Para establecer criterios de ingreso se realizaron consultas individuales de médico, psicólogo y nutricionista. La intervención propiamente tal, tuvo una duración de 4 meses con un control tardío al $6^{\circ}$ mes $^{11}$. Este último fue realizado por nutricionista para evaluar antropometría y hábito de realizar AF. Los controles individuales y talleres grupales se detallan en la Tabla 1 y 2.

\section{Mediciones}

Los pacientes fueron evaluados según peso $(\mathrm{Kg}), \mathrm{IMC}\left(\mathrm{peso} / \mathrm{talla}^{2}\right)$, y CC (cm) al ingreso, al $4^{\circ}$ mes de intervención y en el control tardío del $6^{\circ}$ mes ( 2 meses post-intervención). La medición de peso se realizó en básculas calibradas de pie con tallímetro (precisión de $100 \mathrm{~g}$ y 0,1 cm). Estas medidas fueron utilizadas para calcular el IMC. La CC se midió entre la cresta iliaca y la última costilla con una huincha no elástica (precisión $0,1 \mathrm{~cm}$ ), considerando obesidad abdominal $\geq 88 \mathrm{~cm}$. Igualmente, se evaluó el hábito de realizar AF en tiempo libre, a través de una encuesta estandarizada que consideró frecuencia, duración e intensidad. La glicemia se midió al inicio y al $4^{\circ}$ mes sólo en las alteradas al ingreso $\geq 100 \mathrm{mg} / \mathrm{dL}$. Esta medición fue realizada por profesionales capacitados de cada centro de salud, según normas técnicas. 
Intervención integral en factores de riesgo cardiovascular en mujeres con sobrepeso u obesidad - L. Fuentes L. et al

Tabla 1. Controles individuales

\begin{tabular}{|c|c|c|c|}
\hline Profesional & $\mathrm{N}^{\circ}$ de controles & Tiempo del control & Objetivos \\
\hline Médico & 1 & Ingreso & $\begin{array}{l}\text { Anamnesis, actividad física, uso de medicamentos, ante- } \\
\text { cedentes mórbidos personales y familiares } \\
\text { Evaluación antropométrica y criterios de inclusión } \\
\text { Derivación a psicólogo }\end{array}$ \\
\hline Psicólogo & 1 & Ingreso & $\begin{array}{l}\text { Realizar una entrevista diagnóstica para descartar patolo- } \\
\text { gía psiquiátrica que contraindique el ingreso al programa } \\
\text { Derivación a nutricionista }\end{array}$ \\
\hline Nutricionista & 3 & Ingreso, $4^{\circ}$ y $6^{\circ}$ mes & $\begin{array}{l}\text { Evaluar hábitos de alimentación, actividad física, antropo- } \\
\text { metría (peso, talla, IMC, circunferencia de cintura) } \\
\text { Consejería breve en vida sana } \\
\text { Firma de carta compromiso } \\
\text { Control } 4^{\circ} \text { y } 6^{\circ} \text { mes, evaluar resultados de intervención } \\
\text { alimentaria y de actividad física }\end{array}$ \\
\hline
\end{tabular}

Tabla 2. Talleres Grupales

\begin{tabular}{|c|c|c|}
\hline Profesional & No de sesiones & Objetivos \\
\hline $\begin{array}{l}\text { Profesor de } \\
\text { educación física } \\
\text { o kinesiólogo }\end{array}$ & $\begin{array}{l}32 \text { sesiones, distribuidas a lo largo } \\
\text { de los } 4 \text { meses de intervención, } \\
2 \text { veces por semana, en días no } \\
\text { consecutivos, con una duración de } \\
60 \text { minutos en grupos de máximo } \\
15 \text { personas }\end{array}$ & $\begin{array}{l}\text { Consta de } 3 \text { etapas: } \\
\text { 1.- Fase calentamiento previo ( } 10 \text { minutos) } \\
\text { 2.- Fase fortalecimiento de la capacidad muscular e incremento } \\
\text { de la capacidad aeróbica. Trabajo de } 6 \text { grupos musculares ais- } \\
\text { lados: bíceps, tríceps, abdominales, pectoral, hombros, muslos } \\
\text { y gemelos. Cada grupo muscular se trabaja durante } 1 \text { minuto, } \\
\text { seguido de dos minutos de descanso, con } 3 \text { repeticiones por } \\
\text { grupo muscular ( } 45 \text { minutos). } \\
\text { 3.- Fase relajación y elongación ( } 5 \text { minutos) }\end{array}$ \\
\hline Nutricionista & 8 talleres, una sesión cada 15 días & $\begin{array}{l}\text { Educación en alimentación saludable, asociando conceptos a } \\
\text { decisiones cotidianas y prácticas. Los temas son los siguientes: } \\
\text { - Comer sano y cómo comer } \\
\text { - Mitos relacionados al peso saludable y alimentación } \\
\text { - Guías alimentarias, tamaño de las porciones } \\
\text { - Guías de actividad física } \\
\text { - Etiquetado nutricional } \\
\text { - Colaciones saludables }\end{array}$ \\
\hline Psicólogo & 7 talleres, 1 sesión cada 15 días & $\begin{array}{l}\text { Concientizar al paciente sobre la influencia de sus hábitos de } \\
\text { alimentación y actividad física en su vida diaria, identificando } \\
\text { factores personales o ambientales que influyen en la toma de } \\
\text { decisiones, proporcionando habilidades para generar un cambio. } \\
\text { La estructura temaria de los talleres fue la siguiente: } \\
\text { - "Estamos todos en la misma, iayudémonos a cambiar!" } \\
\text { - "¿Por qué tengo sobrepeso?" } \\
\text { - "¿Cómo me veo y cómo me ven los demás?" } \\
\text { - "Me atrevo a sentir, me atrevo a decir" } \\
\text { - "¿En qué he cambiado y qué me falta por cambiar?" (quinta, } \\
\text { sexta y séptima sesión) }\end{array}$ \\
\hline
\end{tabular}




\section{Análisis de Datos}

Los parámetros registrados (peso, IMC, CC $y$ glicemia), se analizaron en forma general y en función de 3 categorías de edad (basada en una distribución equitativa de la muestra), IMC (sobrepeso y obeso), asistencia a $\mathrm{AF}$ al $4^{\circ}$ mes y hábito de realizar AF al $6^{\circ}$ mes. También se evaluó la asistencia a talleres de nutricionista y psicólogo.

Los datos se procesaron en los programas Microsoft Excel y SPSS 12.0. Se analizó la normalidad de las variables a través de la prueba Shapiro-Wilk. Tanto las diferencias entre evaluaciones, como las diferencias entre sub-grupos de la muestra, se evaluaron a través de las pruebas no paramétricas Mann-Whitney y Kruskal-Wallis. El nivel de confianza utilizado fue de $95 \%$.

\section{Resultados}

\section{Características del grupo estudio}

La Tabla 3 presenta la distribución de las participantes según IMC, categorías de edad y causal de ingreso.

Las pacientes presentaron un promedio de edad de $35 \pm 10,2$ años. El valor promedio de su

\section{Tabla 3. Características de los participantes según estado nutricional, edad y causales de ingreso}

\begin{tabular}{|c|c|c|}
\hline & $\mathbf{N}$ & $\%$ \\
\hline Número de pacientes & 128 & 100 \\
\hline \multicolumn{3}{|l|}{ Patologías (IMC) } \\
\hline Sobrepeso & 35 & 27,3 \\
\hline Obesidad & 93 & 72,6 \\
\hline \multicolumn{3}{|c|}{ Edad (promedio 35 $\pm 10,2$ años) } \\
\hline$<30$ años & 37 & 29 \\
\hline 30 a 40 años & 49 & 38,3 \\
\hline$>40$ años & 42 & 32,8 \\
\hline \multicolumn{3}{|l|}{ Causal de Ingreso } \\
\hline 2 glicemias alteradas & 2 & 1,6 \\
\hline Antecedentes de DM2 & 69 & 53,9 \\
\hline Pre-Hipertensos & 13 & 10,2 \\
\hline 2 o más patologías & 44 & 34,4 \\
\hline
\end{tabular}

peso, IMC y CC al ingreso fue $79,3 \mathrm{~kg}, 32,8 \mathrm{~kg} / \mathrm{m}^{2}$ y $100,8 \mathrm{~cm}$, respectivamente. Se presentó obesidad abdominal en el $90 \%$ de los casos $(\geq 88 \mathrm{~cm})$ y glicemias alteradas en el $29 \%$ de los pacientes. Al final de la intervención ( $4^{\circ}$ mes) estos pacientes disminuyeron su glicemia en $10,2 \mathrm{mg} / \mathrm{dL}(\mathrm{p}<$ $0,001)$, correspondiente a una reducción del 9,2\%.

La medición de la presión arterial no fue considerada dentro del programa en los controles del $4^{\circ}$ y $6^{\circ}$ mes, por lo cual no fue posible identificar si la intervención afectó este parámetro respecto de los valores iniciales.

Como se muestra en la Figura 1, el programa favoreció la reducción de peso, IMC y CC al $4^{\circ}$ y $6^{\circ}$ mes, no obstante ninguna de estas diferencias fue significativa. La reducción porcentual al $4^{\circ}$ mes alcanzó 2,4, 2,1 y 2\% en el peso, IMC y CC, respectivamente. Por su parte, la reducción de parámetros fue superior al $6^{\circ}$ mes, aumentando a $3,2 \%$ en peso, $3 \%$ en IMC y $3,3 \%$ en CC.

Del total de sujetos, $24,2 \%$ bajaron más de $5 \%$ de su peso corporal al $4^{\circ}$ mes (16\% sobrepeso y $84 \%$ obesos), logrando una reducción promedio de $7,3 \pm 4,49 \mathrm{~kg}(\mathrm{p}<0,05)$.

\section{Efectos del programa según categorías de IMC (sobrepeso, obeso) y edad}

Tanto las pacientes con sobrepeso como las obesas redujeron el valor medio de sus parámetros (peso, IMC y CC) después de la intervención, sin alcanzar diferencias significativas al $4^{\circ}$ y $6^{\circ}$ mes. A pesar de esto el $10 \%$ de los pacientes obesos dejaron de serlo, mientras que el $4 \%$ de los pacientes con sobrepeso alcanzaron un estado nutricional normal al cabo del programa.

Según categoría de edad, el peso e IMC mostraron diferencias significativas entre ingreso, $4^{\circ}$ y $6^{\circ}$ mes ( $p<0,05)$, principalmente entre los $<30$ y los $>40$ años, donde la mayor disminución ocurrió en los $>40$ años al $6^{\circ}$ mes, con $-4,51 \mathrm{~kg}$ de peso, $-1,91$ $\mathrm{kg} / \mathrm{m}^{2}$ de IMC y $-4,3 \mathrm{~cm}$ de CC. Esto también se observó al distribuir la muestra según categorías de edad e IMC, donde las mayores diferencias significativas tanto en los sobrepeso como en los obesos también ocurrieron entre los $\langle 30$ y $\rangle 40$ años, representado en la Figura 2. En este caso, las mayores reducciones se registraron al $6^{\circ}$ mes en los obesos > 40 años (-5,02 kg de peso, $-2,08 \mathrm{~kg} /$ $\mathrm{m}^{2}$ de IMC y $-4,4 \mathrm{~cm}$ de CC).

No se encontraron diferencias significativas en los niveles de glicemia entre ingreso y $4^{\circ}$ mes según 




Figura 1. Valor medio del peso, IMC y CC en controles de ingreso, $4^{\circ}$ y $6^{\circ}$ mes. En el eje izquierdo de las ordenadas se presenta la escala del peso y CC, y en el lado derecho de este eje la escala del IMC.

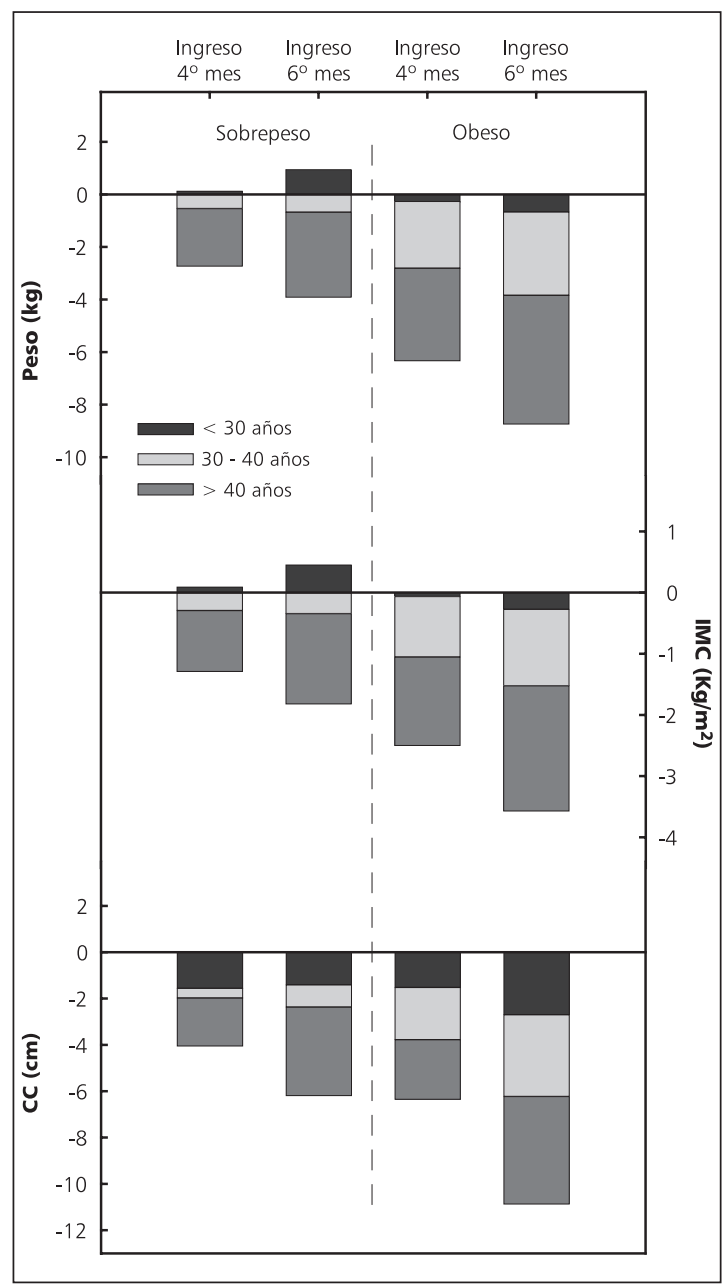

Figura 2. Diferencias de parámetros al ingreso, $4^{\circ}$ y $6^{\circ}$ mes, según categoría de edad y grupos de Sobrepeso y Obesos. categorías de edad e IMC. Sin embargo, siguiendo la misma tendencia de los parámetros antropométricos, la glicemia presentó mayor disminución en los $>40$ años $(-15,4 \mathrm{mg} / \mathrm{dL})$.

\section{Efectos del programa según práctica de actividad física (AF) al $4^{\circ}$ y $6^{\circ}$ mes}

Para evaluar los efectos de la asistencia a las sesiones de AF durante los 4 meses de intervención, ésta se categorizó en rangos que van de $0-25 \%, 26-50 \%, 51-75 \%$ y $76-100 \%$. De este modo se determinó que 21 pacientes asistieron entre $0-25 \%, 22$ entre $26-50 \%, 27$ entre $51-75 \%$ y 58 al $76-100 \%$.

En la Figura 3 se observa que la asistencia a sesiones de AF tuvo un efecto significativo en la reducción de peso, IMC y CC entre grupos ( $\mathrm{p}<$ $0,05)$. La principal reducción de peso $(-3,19 \mathrm{~kg})$, IMC $\left(-1,3 \mathrm{~kg} / \mathrm{m}^{2}\right)$ y CC $(-2,9 \mathrm{~cm})$, se presentó en los asistentes al 76-100\%. No obstante, la AF no influyó significativamente en los niveles de glicemia.

En la Tabla 4 se puede observar que la mayor asistencia a sesiones de AF ocurrió en los pacientes con sobrepeso $>40$ años, seguidos por los obesos entre 30-40 años y los $>40$ años.

$\mathrm{Al}$ inicio del programa el 84\% de los pacientes no realizaban AF en tiempo libre. Sin embargo, en el control del $6^{\circ}$ mes el 59\% desarrollaba AF (77\% sobrepeso y $50 \%$ obesos), de los cuales el $85 \%$ realizaba $\mathrm{AF} 3$ a 4 días a la semana, de moderada intensidad, durante 45 minutos o más.

Se encontraron diferencias significativas en peso e IMC entre el grupo que realizaba $\mathrm{AF}$ y el que no practicaba $\mathrm{AF}$ al $6^{\circ}$ mes $(\mathrm{p}<0,05)$. La reducción de parámetros del grupo que realizaba $\mathrm{AF}$ al $6^{\circ}$ mes, fue de $-4,65 \pm 3,97 \mathrm{~kg}$ en peso, $-1,95 \pm$ $1,64 \mathrm{~kg} / \mathrm{m}^{2}$ en IMC y $-7,12 \pm 6,72 \mathrm{~cm}$ en CC. Esto significó una disminución de $26 \%$ en peso, $23 \%$ en IMC y $25 \%$ en CC, respecto del grupo que no realizó $\mathrm{AF}$ al $6^{\circ}$ mes.

\section{Asistencia a talleres de nutricionista y psicólogo}

La asistencia promedio del grupo a talleres de nutricionista fue de $4,3(54 \%)$ y 1,8 a talleres de psicólogo (26\%).

Según categorías de edad e IMC, tanto los sobrepeso como los obesos $>40$ años, asistieron en mayor cantidad a (> 75\%) talleres de nutricionista, como se muestra en la Tabla 5. En cambio, la asistencia a talleres de psicólogo en todas las categorías fue baja. 
Tabla 4. Porcentaje de asistencia a AF según categorías de edad e IMC

\begin{tabular}{|rccccccc|}
\hline \% asistencia AF & \multicolumn{3}{c}{ Sobrepeso } & \multicolumn{3}{c|}{ Obesos } \\
& $<\mathbf{3 0}$ años & $\mathbf{3 0 - 4 0}$ años & $\mathbf{>} \mathbf{4 0}$ años & $<\mathbf{3 0}$ años & $\mathbf{3 0 - 4 0}$ años & $>\mathbf{4 0}$ años \\
\hline $0-25$ & 30 & 23,1 & 8,3 & 22,2 & 11,1 & 13,3 \\
\hline $26-50$ & 10 & 23,1 & 16,7 & 14,8 & 19,4 & 16,7 \\
\hline $51-75$ & 30 & 15,4 & 8,3 & 29,6 & 13,9 & 26,7 \\
$76-100$ & 30 & 38,5 & 66,7 & 33,3 & 55,6 & 43,3 \\
\hline
\end{tabular}

Tabla 5. Asistencia a talleres de nutricionista según categorías de edad e IMC

\begin{tabular}{|rcccccc|}
\hline $\begin{array}{l}\text { \% asistencia } \\
\text { talleres } \\
\text { nutricionista }\end{array}$ & $\mathbf{<}$ años & $\begin{array}{c}\text { Sobrepesos } \\
\mathbf{3 0 - 4 0} \text { años }\end{array}$ & $\boldsymbol{>} \mathbf{4 0}$ años & $\mathbf{<} \mathbf{3 0}$ años & $\mathbf{3 0 - 4 0}$ años & $>\mathbf{4 0}$ años \\
\hline $0-25$ & 50 & 38,5 & 41,7 & 40,7 & 33,3 & 41,7 \\
\hline $26-50$ & - & 23,1 & - & 29,6 & 27,8 & - \\
\hline $51-75$ & 10 & 7,7 & - & 18,5 & 8,3 & - \\
$76-100$ & 40 & 30,8 & 58,3 & 11,1 & 30,6 & 58,3 \\
\hline
\end{tabular}

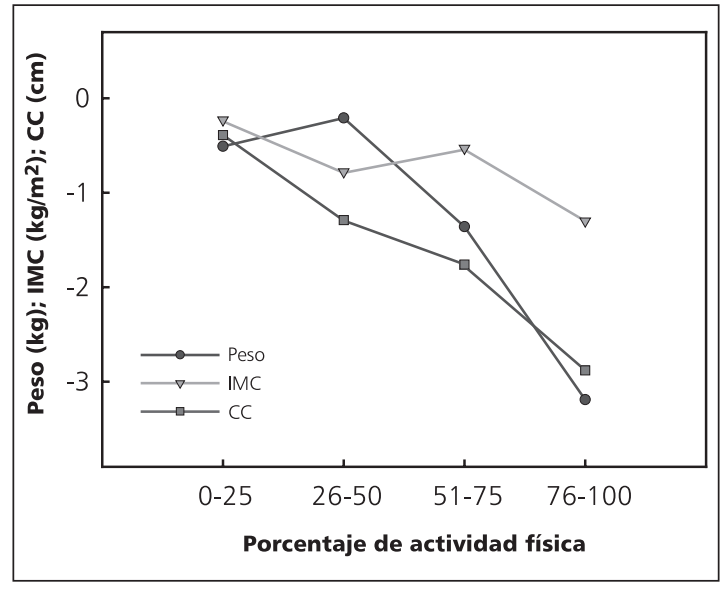

Figura 3. Asistencia a sesiones de Actividad Física (AF) al $4^{\circ}$ mes.

\section{Discusión}

\section{Efectividad del programa sobre el grupo estudiado}

A pesar de no encontrarse una reducción significativa de peso, IMC y CC en el grupo general y en los sub-grupos de sobrepeso y obesos, la intervención es considerada efectiva, ya que la sola detención del aumento de peso en el grupo constituye un éxito en la prevención de enfermedades cardiovasculares ${ }^{12}$. Conjuntamente, la promoción de estilos de vida saludables en la población, asociada a la práctica regular de $\mathrm{AF}$ al $6^{\circ}$ mes, fue considerada efectiva en más de la mitad de los participantes (59\%). Este grupo realizó en su mayoría (85\%) AF en forma moderada, presentando diferencias significativas en sus parámetros respecto del grupo que no continuó realizando ejercicio. Esto confirma que una AF moderada es efectiva en la reducción de peso y riesgos cardiovasculares $^{13,14}$.

A diferencia del presente estudio en el que sólo $24,2 \%$ bajaron más de $5 \%$ de su peso al $4^{\circ}$ mes de intervención, una evaluación nacional del programa en el año 2007 reveló que 40,1\% de los participantes logró dicho objetivo ${ }^{15}$.

En general, en las evaluaciones realizadas en el presente estudio, la reducción de los parámetros antropométricos fue mayor al $6^{\circ}$ mes, debido posiblemente a un mayor tiempo practicando AF y al aprendizaje alimentario adquirido ${ }^{16}$.

Los pacientes con glicemia alterada al ingreso presentaron una reducción significativa al $4^{\circ}$ mes, independiente que la reducción general de parámetros no alcanzara valores significativos. 
Esto revela que es posible reducir la glicemia con niveles moderados de $\mathrm{AF}^{9,10,17,18}$, sin coexistir necesariamente una reducción significativa de peso, IMC y CC.

\section{Efectos del programa según edad e IMC}

(sobrepeso, obeso)

Diversos estudios afirman que cambios en la alimentación junto a la práctica de $\mathrm{AF}$ son indispensables en el tratamiento del sobrepeso y la obesidad ${ }^{11,15}$,y que este proceso es más efectivo acompañado de terapia psicológica ${ }^{19}$. En este sentido y según la Encuesta de Calidad de Vida y Salud 2006, los > 40 años tienen mejores hábitos alimentarios que los $<20$ años $^{3}$, relacionado con el consumo de fibra, lácteos, comida chatarra, consumo de desayuno, etc. Lo anterior puede explicar en gran parte la mayor efectividad del programa en este grupo etáreo. Igualmente, el compromiso respecto de la intervención, la motivación interna, la responsabilidad y una mayor madurez psicológica puede influir significativamente en el éxito y la adherencia a la intervención ${ }^{20}$.

Según edad y sub-grupos de sobrepeso y obesos, las mayores reducciones que alcanzaron niveles significativos se observaron en la evaluación del $6^{\circ}$ mes en pacientes obesos $>40$ años. Dicho efecto se puede asociar al mayor porcentaje de asistencia a actividades del programa (sesiones de AF y talleres) $)^{21,22}$ y al porcentaje de pacientes obesos $>40$ años que continúo realizando $\mathrm{AF}$ al $6^{\circ}$ mes $(58,1 \%)$.

Al igual que los resultados principales, los $>40$ años lograron una mayor disminución de glicemia, alcanzando niveles normales en el 13,5\% de los pacientes que tuvieron este parámetro alterado al ingreso.

\section{Consideraciones para el mejoramiento del programa}

Si bien el programa logró su objetivo principal sólo en $24 \%$ de las pacientes -reducir al menos el $5 \%$ del peso, IMC y CC- este estudio permite identificar posibles causas asociadas. Por ejemplo, es relevante mejorar la asistencia a los talleres de especialistas y de AF, ya que estos se relacionaron con una mayor efectividad del programa. Cabe mencionar que un monitoreo dietario permitiría mejorar la relación dieta-AF, considerada clave para la eficiencia de programas interdisciplina- $\operatorname{rios}^{21,23}$. De igual manera, es necesario incluir la satisfacción usuaria a fin de considerar cambios locales dentro del programa. También pueden ser incorporados en las evaluaciones registros sobre los beneficios psicológicos atribuibles a la práctica regular de AF, los cuales han sido relacionados con el grado de estrés y estado de ánimo ${ }^{24}$.

Asimismo, es necesario aumentar la eficiencia de las intervenciones de promoción y centrar la investigación en una mejor comprensión de los predictores que influyen en el desarrollo de AF en la población objetivo ${ }^{25}$. Respecto de este punto, son factores importantes para el desarrollo de AF, el ambiente y el espacio urbano disponible para su desarrollo ${ }^{26}$.

\section{Agradecimientos}

A los equipos de salud que ejecutaron el programa en los distintos centros asistenciales de la Región de Los Ríos. Al Sr. E. Athala y a dos revisores anónimos por sus valiosos comentarios durante la preparación de este artículo.

\section{Referencias}

1. Albala C, Kain J, Burrows R, Díaz E. Obesidad: un desafío pendiente. Albala C, Kain J, Burrows R, Díaz E, editores. Santiago, Chile: Editorial Universitaria, Vicerrectoría de Asuntos Académicos Universidad de Chile 2000; p 31-43.

2. Ministerio de Salud. Encuesta de Salud, 2003. Minsal, Chile. Disponible en: http://epi.minsal.cl/epi/html/elvigia/VIGIA20.pdf (Consultado el 13 de abril de 2009).

3. Ministerio de Salud, SubSecretaría de Salud Pública, División de Planificación sanitaria. Encuesta de Calidad de Vida, 2006. Minsal, Chile. Disponible en: http://epi. minsal.cl/epi/html/sdesalud/calidaddevida2006/Informe\%20Final\%20Encuesta\%20de\%20Calidad\%20de $\% 20$ Vida\%20y\%20Salud\%202006.pdf (Consultado el 22 de abril del 2009).

4. Joint WHO/FAO Expert Consultation. Diet, Nutrition and the Prevention of Chronic Diseases. Report of a Joint. World Health Organitation, Géneva 2003; p 4-8. http://www.who.int/hpr/NPH/docs/who_fao_expert_report.pdf (Consultado 27 de abril de 2009).

5. Salinas J, Vio F. Promoción de la Salud y Actividad Física en Chile: Política Prioritaria. Rev Panam Salud Pública 2003; 14: 281-8. 
6. Ministerio de Salud. Objetivos Sanitarios y Modelo de Salud para la Década 2000-2010, 2002. Minsal, Chile. http://www.navarro.cl/defensa/salud/Doc/Objetivossanitarios2000-2010.pdf (Consultado 1 de mayo de 2009).

7. Vio F. Prevención de la Obesidad en Chile. Rev Chil Nutr 2005; 32: 80-7.

8. Scarsella C, Després JP. Tratamiento de la Obesidad: necesidad de centrar la atención en los pacientes de alto riesgo caracterizados por la obesidad abdominal. Cad. Saûde Pública, Río de Janeiro 19, 2003; 1: 7-19.

9. Hu F, Manson J, Stamfer M, Colditz G, Liu S, Solomon C, et al. Diet, Lifestyle, and the Risk of Type 2 Diabetes Mellitus in Woman. N Engl J Med 2001; 345: 790-97.

10. Tuomilehto J, Lindstrom J, Eriksson J, Valle T, Hamalainen $\mathrm{H}$, Illanne-Parikka P, et al. Prevention of Type 2 Diabetes Mellitus by Changes in Lifestyle among Subjects with Impaired Glucose Tolerance. N Engl J Med 2001; 344: 1343-50.

11. Carrasco F, Moreno M, Irribarra V, Rodríguez L, Martin MA, Alarcón A, et al. Evaluación de un programa piloto de intervención en adultos con sobrepeso u obesidad, en riesgo de diabetes. Rev Med Chile 2008; 136: 13-21.

12. WHO Obesity. Preventing and managing the global epidemic. Report of a WHO. Consultation on Obesity. Geneva (Consultado 23 de abril de 2009).

13. Pratt M. Benefits of Lifestyle Activity vs Structured Exercise. JAMA 1999; 281:375-376.

14. Haskell W, Lee I, Pate R, Powell K, Blair S, Franklin B, et al. Physical activity and public health: updated recommendation for adults from the American College of Sports Medicine and the American Heart Association. JAMA 1995; 273: 402-7.

15. Gobierno de Chile, Ministerio de Hacienda, Dirección de Presupuestos. Minuta Ejecutiva, Programa de Tratamiento de la Obesidad "Alimentación Saludable y Actividad Física (en Adultos, Niños y adolescentes), 2008. http://www.dipres.cl/574/articles-38659_doc_pdf.pdf. (Consultado 21 de mayo de 2009).

16. Moreno M, Manrique M, Guzmán S, Maiz A, Patiño C, Valdés R, et al. Cambios en los factores de riesgo meta- bólicos en pacientes obesos en tratamiento. Rev Med Chile 2000; 128; 193-200.

17. Kirk A, Mutrie N, Maclntyre P, Fisher M. Increasing Physical Activity in People With Type 2 Diabetes. Diabetes Care 2003; 26: 1186-92.

18. Bassukl S, Manson J. Epidemiological evidence for the role of physical activity in reducing risk of type 2 diabetes and cardiovascular disease. J Appl Physiol 2005; 99: 1193-204.

19. Shaw K, O’Rourke P, Del Mar C, Kenardy J. Psychological interventions for overweight or obesity. The Cochrane Library, 2004 Issue 1.

20. Elfhag K, Rössner S. Who succeeds in maintaining weight loss? A conceptual review of factors associated with weight loss maintenance and weight regain. Obes Rev 2005; 6: 67-85

21. OPS-OMS. Obesidad, alimentación y actividad física. 37. a Sesión del Subcomité de Planificación y Programación del Comité Ejecutivo. Washington, D.C., EUA, 26 al 28 de marzo de 2003:5 http://www.paho.org/spanish/gov/ce/ spp/spp37-08-s.pdf. (Consultado 15 de mayo de 2009).

22. World Health Organization Global strategy on diet, physical activity and health. The world health report. Reducing risks, promoting healthy life. Geneva, 2002.

23. Andersen R, Wadden T, Bartlett S, Zemel B, Verde T, Franckowiak S. Effects of Lifestyle Activity vs Structured Aerobic Exercise in Obese Women. JAMA 1999; 281: $335-40$

24. Jiménez M, Martínez P, Miró E, Sánchez A. Bienestar psicólogico y hábitos saludables: ¿están asociados a la práctica de ejercicio físico? Int J Clin Health Psychol 2008; 8: 185-202.

25. Baranowski T, Anderson C, Carmack C. Mediating variable framework in physical activity interventions. How are we doing? How might we do better? Am J Prev Med 1998; 15: 266-97.

26. Ewing R, Schmid T, Killingsworth R, Zlot A, Raudenbush S. Relationship between urban sprawl and physical activity, obesity, and morbidity. Am J Health Promot 2003; 18: 47-57. 